\title{
A Wearable Inductor Channel Design for Blood Pressure Monitoring System in Daily Life
}

\author{
Seulki Lee, Jerald Yoo, and Hoi-Jun Yoo \\ Department of EECS \\ Korea Advanced Institute of Science and Technology (KAIST) \\ Daejeon, Republic of Korea \\ sklee@eeinfo.kaist.ac.kr
}

\begin{abstract}
This paper proposes a wearable inductor channel for blood pressure monitoring system in daily life. To achieve high wearability and low power consumption, inductive coupling concept with textile-based inductors is introduced. Two types of wearable inductors are proposed, and both of them are demonstrated to have the possibility as a wearable inductor channel for blood pressure sensors in daily life.
\end{abstract}

Keywords-blood pressure monitoring; inductive coupling; near field communication; wearable inductor;

\section{INTRODUCTION}

The greater demand for continuous healthcare monitoring at home instead of at hospitals shows up in these days. Diseases like hypertension or atherosclerosis need to monitor one's health continuously. Since they are not terminal diseases, the patients do not need to stay in the hospital, but have to check frequently their own body temperature, blood pressure, pulse rate, or electrocardiogram (ECG) signal to prevent the progress of the disease. They try to gather these bio-signals in their everyday lives and consult a doctor on their states of health just once in a while. For this reason, many ongoing researches try to achieve wearable sensing, transmission, and processing devices which are suitable for person's daily life [1] - [3]. As shown in Figure 1, the home-care system consists of several sensors and a portable unit (Base station). Among several sensors, the wearable interfaces of ECG, body temperature, and pulse rate sensor have been studied. However, the wearable blood pressure sensor interface has not been studied yet. Although RS-232 connection is used to transmit the bio-signal data including blood pressure to the portable unit [4]-[5], it is not suitable for daily life since it disturbs user's activity. And some researches try to use Bluetooth as a wireless interface for blood pressure sensor [6], but it consumes much more power than measurement operation itself so that it dramatically reduces the battery life. Therefore, a new interface with high wearability and low power consumption is needed for blood pressure monitoring system in daily life. Near Field Communication (NFC) technology based on inductive coupling is considered as a high potential technology for low-power short-range connectivity in these days [7], but the inductive coupling channel of NFC still has a wearability issue which is not solved yet.
In this paper, we propose a wearable inductor channel for the interface of blood pressure monitoring system in daily life. The inductors are made by two ways - one is woven inductor of conductive yarn [2], and the other is silkscreen inductor [8]. Since the substrates of both inductors are same as clothes, they are suitable for high wearability in daily life. The rest of the paper is organized as follows. In Section II, the design of wearable inductor and inductive coupling channel will be covered. Section III describes the dedicated inductive coupling channel for blood pressure sensors and the system integration. Finally, conclusions will be made in Section IV.

\section{WEARABLE INDUCTOR CHANNEL}

\section{A. Woven Inductor}

The woven inductors are made by sewing conductive yarn on a textile as shown in Figure 2. This conductive yarn is already introduced in the previous work [2]. This yarn is coated by polyester, and its thickness is about $100 \mu \mathrm{m}$. It successfully passes washing tests several tens of times. So it can be

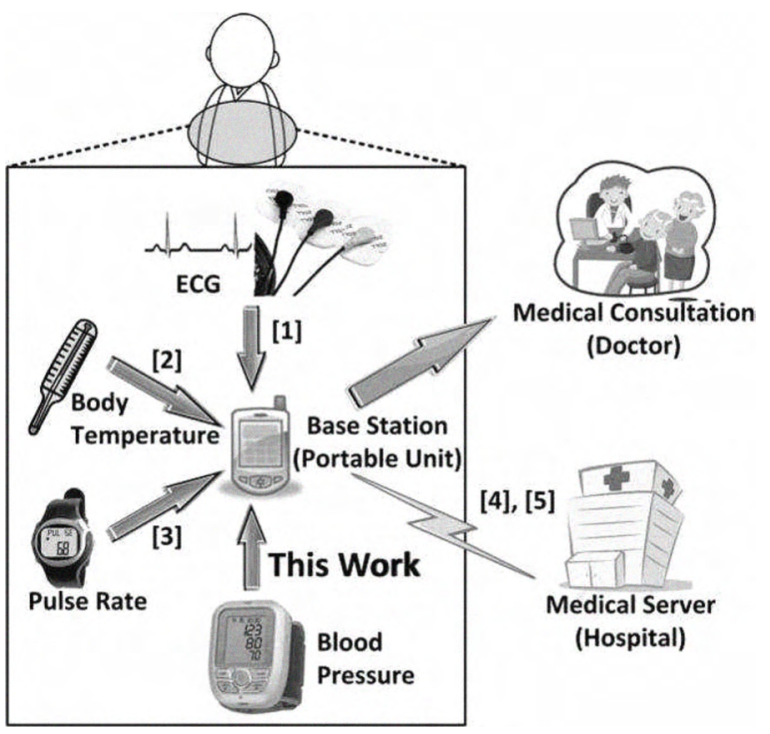

Figure 1. Home-care system architecture 
integrated into clothes perfectly while the clothes are made in the factory. One of the most strong points of this conductive yarn and the woven inductor is that it is suitable for implementing wireline network and also easy to connect wireline network and inductor channels.

As shown in Figure 3, when more than two inductors are closely placed, the magnetic field of one inductor induces the current flow of the other inductors. This is the concept of inductive coupling. In this case, the induced current magnitudes of the other inductors are changed according to the number of turn ratio and position relationship of the corresponding inductors. To clarify the effect of the number of turn ratio and position relationship on the induced current magnitude, we define the channel gain as a ratio of the received voltage magnitude over the transmitted voltage magnitude. For woven inductors, the relationship between gain and the number of turn ratio is represented as in (1):

$$
\text { Gain } \propto \frac{\mathrm{N}_{\mathrm{RX}}}{\mathrm{N}_{\mathrm{TX}}}
$$

where, $\mathrm{N}_{\mathrm{RX}}$ is the number of turns of the receiver inductor and $\mathrm{N}_{\text {TX }}$ is the number of turns of the transmitter inductor [2]. And the relationships between gain and position of two inductors are measured as shown in Figure 4. The square inductors 10$\mathrm{mm}$ on a side with 10-turn are used for measurements. According to the measurements, the distance between two inductors becomes longer, and then the gain becomes smaller. Also, if the inductors are not aligned with each other, the gain becomes smaller. Since the length of each side of the inductor is $10-\mathrm{mm}$, gain of horizontally or vertically misaligned case is smaller than that of the diagonally misaligned case over $10-\mathrm{mm}$ misalignment. From these measurements, the fact that gain of woven inductor channel is related on the cross-sectional area
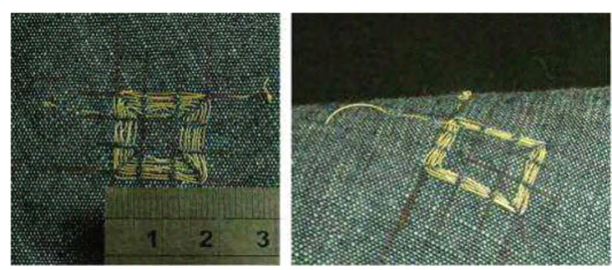

Figure 2. Woven inductors

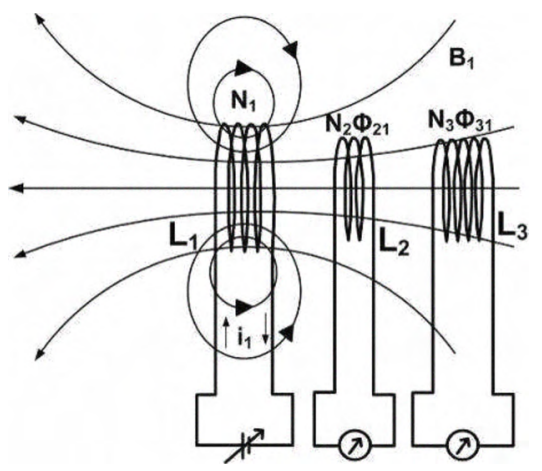

Figure 3. Concept of inductive coupling

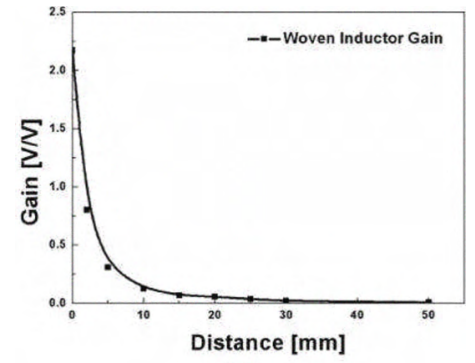

(a)

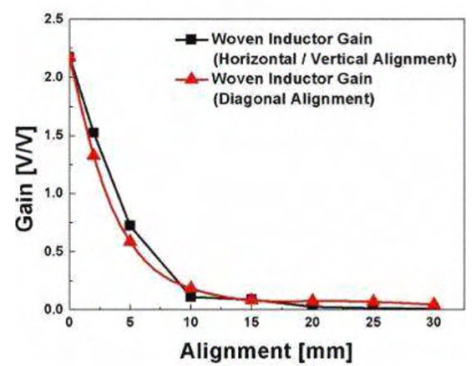

(b)

Figure 4. Gain vs. (a) distance (b) alignment in woven inductor channel
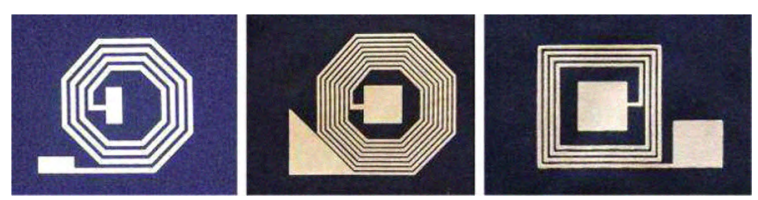

Figure 5. Silkscreen inductors

between two inductors is revealed.

\section{B. Silkscreen Inductor}

The silkscreen inductors are made by silkscreen printing method which is introduced in [8]. The silkscreen printing on a textile uses a conductive ink to form electronic components and circuits. The conductive ink is painted through the open areas of a mesh-reinforced stencil onto the textile. After printing process, the firing process is performed below $150^{\circ} \mathrm{C}$ for $10-$ 30 minutes to avoid the deformation of the textile substrate. The minimum resolution of the silkscreen printing is $200 \mu \mathrm{m}$, and the thickness of the conductive ink is $10 \mu \mathrm{m}$. It also passes washing tests successfully for several tens of times. The silkscreen inductors with a variety of shapes are shown in Figure 5. One merit of silkscreen printing method is that it can make the same components or circuits repeatedly, resulting in low cost and high productivity.

To verify the possibility as a wearable inductor channel, some measurements about inductive coupling of silkscreen inductors were performed. As shown in Figure 6, the gain of silkscreen inductor channel is changed according to the distance and alignment of two inductors. The octagon inductors 30-mm on an outermost side with 8-turn are used for measurements. This inductor has $2 \mu \mathrm{H}$ inductance on average, which is similar value to the woven inductor in Section II-A. 
However, by comparing Figure 4 (a) and Figure 6 (a), the gain of silkscreen inductor channel is smaller than that of the woven inductor channel. This difference is caused by the number of turn ratio between the transmitter inductor and the receiver

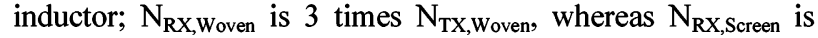
equal as $\mathrm{N}_{\mathrm{TX} \text {,Screen }}$. For silkscreen inductor channel, the maximum number of turns for an inductor is limited by the physical size of it since each line cannot be overlapped each other. To solve this problem, the multi-layer inductor structure is proposed in this work which will be described in section III. According to the measurement in Figure 6 (b), the gain of horizontally or vertically misaligned case and diagonally misaligned case is similar. This is because the shape of the inductor is octagon which is almost same as circle, so the radius in any direction is very similar.

\section{Comparison}

In Section II, two types of the wearable inductor were shown. Table I shows the comparison between the woven inductor and the silkscreen inductor in terms of DC resistance,

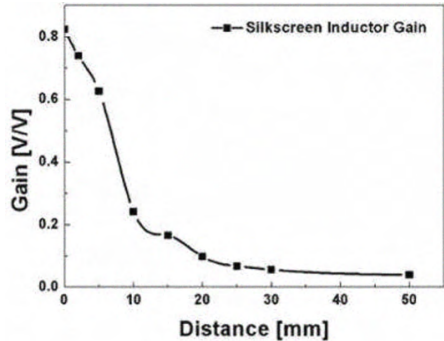

(a)

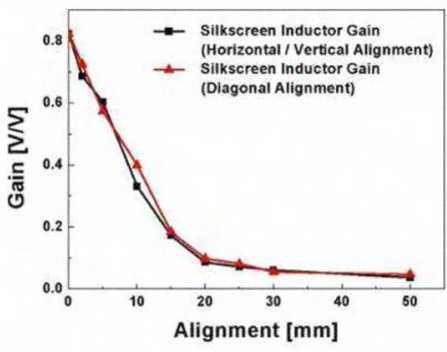

(b)

Figure 6. Gain vs. (a) distance (b) alignment in silkscreen inductor channel

TABLE I. COMPARISON BETWEEN TWO TYPES OF INDUCTORS

\begin{tabular}{|c|c|c|}
\hline & Woven inductor & Silkscreen Inductor \\
\hline DC Resistance & $6 \Omega / \mathrm{m}$ & $13.35 \Omega / \mathrm{m}$ \\
\hline Inductance* & $2.3 \mu \mathrm{H}$ & $2 \mu \mathrm{H}$ \\
\hline Durability & High & High \\
\hline Wearability & High & High \\
\hline Mass production & Hard & Easy \\
\hline
\end{tabular}

* Inductance is measured using the inductors in section II. inductance, durability, wearability and mass production. As shown in Table I, the electrical characteristic like DC resistance or inductance of the woven inductor is little bit better than the silkscreen inductor. However, the productivity of the silkscreen inductor is much better than the woven inductor. Both types of inductors are possible as a wearable inductor channel on a case by case basis.

\section{DEDICATED CHANNEL FOR BLOOD PRESSURE SENSORS}

\section{A. Multi-layer Inductor Structure}

In consideration of the forearm size, the typical dimension of the wearable device on the forearm would be smaller than 60 $x 50 \mathrm{~mm}^{2}$ [9]. In case of silkscreen inductors with this size, the inductance is limited to $3.2 \mu \mathrm{H}$. In some cases such that the distance between inductors is increased than usual, the receiver inductor may need larger inductance to compensate the gain. As shown in Figure 7, the multi-layer inductor structure is proposed in this paper to increase the inductance of the inductor without any physical size extension. In this structure, more than two silkscreen inductors are piled up, and they are connected to each other with same current direction. The measurement result of multi-layer inductor structure is shown in figure 8. The impedance, i.e. inductance, is proportional to the number of inductor layers, and the self-resonant frequency is inversely proportional to it. Hence the gain of channel is enhanced when the number of layer for the receiver inductor is increased. Since the data rate of blood pressure sensors are below a few kbps, the reduction of self-resonant frequency

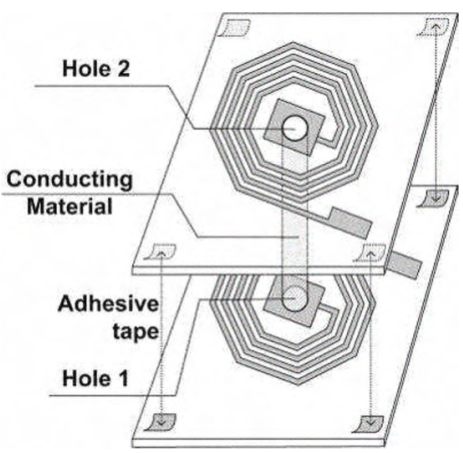

Figure 7. Multi-layer inductor structure

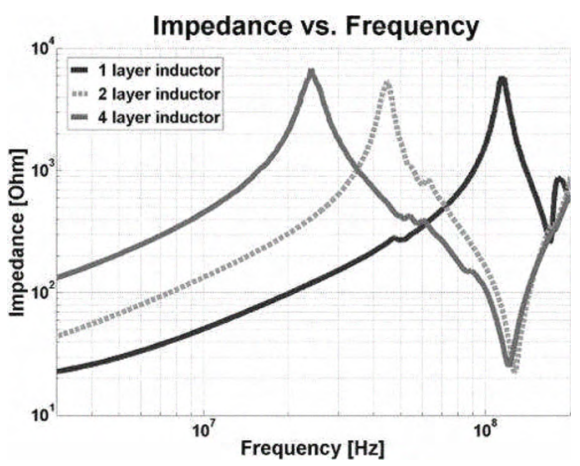

Figure 8. Frequency characteristic for variety of inductor layers 
does not affect the data transmission.

\section{B. Bending Effects}

In case that the physical size of the inductor is similar to the maximum area of a forearm, the fabric substrate of the inductor is bent so that the inductance and the gain are changed. Therefore, the variance of the gain should be checked in order to apply this channel to the blood pressure monitoring system. Figure 9 shows the bending effect of the channel. Each type of the inductor is used to measure the channel gain in case that the inductor is bent in several forms. The channel gains are decreased for both cases, but the extent of the gain reduction can be compensated by increasing the inductance of the receiver as shown in Figure 8.

\section{System Integration}

System integration between the channel and the blood pressure sensor is shown in Figure 10. The transmitter inductor

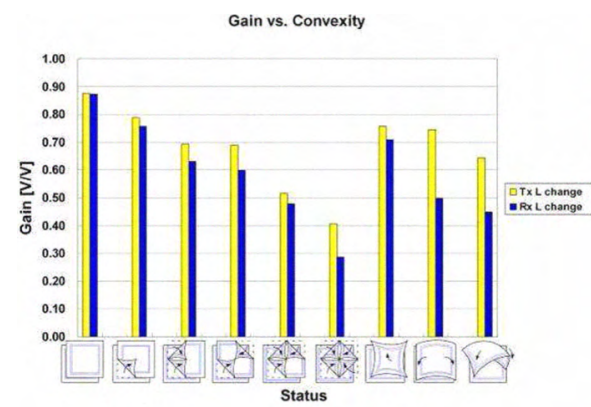

(a)

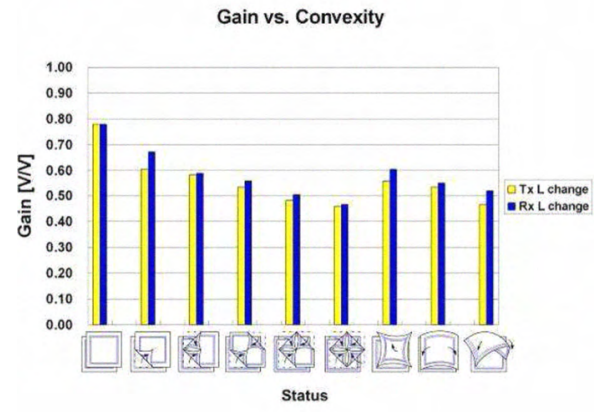

(b)

Figure 9. Gain vs. convexity (a) woven inductor channel (b) silkscreen inductor channel

Wearable Inductor (RX) : Attach to clothes

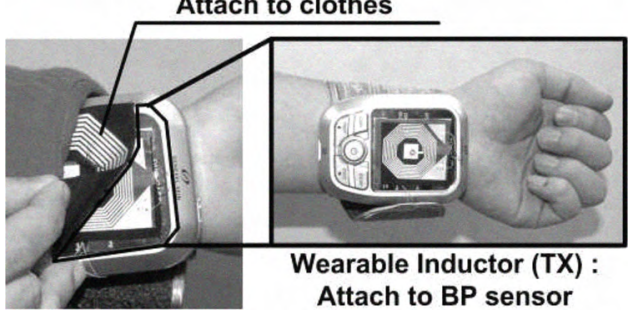

Figure 10. Blood pressure sensor and wearable channel integration is attached on the blood pressure sensor and the receiver inductor is placed at the clothes. By putting on the blood pressure sensor on a wrist and measuring as usual, the blood pressure data can be transmitted to the portable unit (Base station) which is placed on the pocket of the jacket. The system can achieve high wearability and low power consumption, so it is very suitable for blood pressure monitoring in daily life.

\section{CONCLUSION}

Two types of wearable inductor channels for blood pressure monitoring system in daily life are proposed and developed. To maximize the wearability and achieve low power consumption, inductive coupling concept with textile-based inductors is introduced. And the manufacture process and channel gain characteristics of each type of inductors are explained. Both woven inductors and silkscreen inductors show high wearability so that they are suitable for blood pressure monitoring in daily life. These channels can be expected to function effectively when a total blood pressure monitoring system is developed in the near future.

\section{REFERENCES}

[1] Jerald Yoo, Long Yan, Seulki Lee, Yongsang Kim, Hyejung Kim, Binhee Kim, and Hoi-Jun Yoo, "A 5.2mW Self-Configured Wearable Body Sensor Network Controller and a 12uW 54.9\% Efficiency Wirelessly Powered Sensor for Continuous Health Monitoring System," IEEE ISSCC Digest Technical Papers, pp.290-291, Feb., 2009.

[2] Seulki Lee, Jerald Yoo, and Hoi-Jun Yoo, "A Healthcare Monitoring System with Wireless Woven Inductor Channels for Body Sensor Network," Proceedings of the $5^{\text {th }}$ International Workshop on Wearable and Implantable Body Sensor Networks (BSN), pp.62-65, 2008.

[3] Xin Zhu, Wenxi Chen, Tetsu Nemoto, Yumi Kanemitsu, Kei-ichiro Kitamura, Ken-ichi Yamakoshi, and Daming Wei, "Real-Time Monitoring of Respiration Rhythm and Pulse Rate During Sleep," IEEE Transactions on Biomedical Engineering, Vol.53, No.12, pp.2553-2563, Dec., 2006.

[4] Sotiris Pavlopoulos, Efthyvoulos Kyriacou, Alexis Berler, Spyros Dembeyiotis, and Dimitris Koutsouris, "A Novel Emergency Telemedicine System Based on Wireless Communication Technology AMBULANCE," IEEE Transactions on Information Technology in Biomedicine, Vol. 2, No. 4, Dec., 1998.

[5] Yuan-Hsiang Lin, I-Chien Jan, Patrick Chow-In Ko, Yen-Yu Chen, JauMin Wong, and Gwo-Jen Jan, "A Wireless PDA-Based Physiological Monitoring System for Patient Transport" IEEE Transactions on Information Technology in Biomedicine, Vol. 8, No. 4, Dec., 2004.

[6] Ren-Guey Lee, Chun-Chieh Hsiao, Chun-Chung Chen, and Ming-Shiu Liu, "A Mobile-Care System Integrated with Bluetooth Blood Pressure and Pulse Monitor, and Cellular Phone," IEICE Transaction on Information and Systems, Vol.E89-D, No.5, pp.1702-1711, May, 2006.

[7] Esko Strömmer, Jouni Kaartinen, Juha Pärkkä, Artoo Ylisaukko-oja, and Ilkka Korhonen, "Application of Near Field Communication for Health Monitoring in Daily Life," Proceedings of the $28^{\text {th }}$ IEEE EMBS Annual International Conference, pp. 3246-3249, Sep., 2006.

[8] Yongsang Kim, Hyejung Kim, and Hoi-Jun Yoo, "Electrical Characterization of Printed Circuits on the Fabric," IEEE Transactions on Advanced Packaging. (Submitted)

[9] M. Scheffler and E. Hirt, "Wearable devices for telemedicine applications," Journal of Telemedicine and Telecare, Vol.11, pp.11-14, 2005. 